\title{
Resonant Drift of Spiral Waves in the Complex Ginzburg-Landau Equation
}

\author{
IRINA V. BIKTASHEVA*, YURY E. ELKIN and VADIM N. BIKTASHEV** \\ Institute for Mathematical Problems in Biology, 142292 Pushchino, Moscow region, Russia \\ * Current address: School of Biomedical Sciences, University of Leeds, Leeds LS2 9JT, UK \\ ** Current address: School of Biomedical Sciences, University of Leeds, Leeds LS2 9JT, UK
}

Accepted in final form 29 January 1999

\begin{abstract}
Weak periodic external perturbations of an autowave medium can cause large-distance directed motion of the spiral wave. This happens when the period of the perturbation coincides with, or is close to the rotation period of a spiral wave, or its multiple. Such motion is called resonant or parametric drift. It may be used for low-voltage defibrillation of heart tissue. Theory of the resonant drift exists, but so far was used only qualitatively. In this paper, we show good quantitative agreement of the theory with direct numerical simulations. This is done for Complex Ginzburg-Landau Equation, one of the simplest autowave models.
\end{abstract}

Key words: Response functions, Resonant drift, Parametric drift, Asymptotics, Spiral waves, Oscillatory medium, Autowaves, Reaction-diffusion System.

\section{Introduction}

Spiral waves are a specific form of self-organisation observed in various biological systems; of the most practical importance are those in the cardiac muscle, where spiral waves underlie dangerous arrhythmias including fibrillation [1]. The dynamics of spiral waves in a slightly perturbed two-dimensional autowave media (e.g. slightly inhomogeneous or subject to slight external forcing), modelled by 'reaction+diffusion' systems of partial differential equations, can be described asymptotically in terms of 'Aristotelean' dynamics, so that the velocities of the spiral wave drift in space and time are proportional to the 'forces' caused by the perturbation. These forces are defined as convolutions of the perturbation with the sensitivity (response) functions [2, 3].

One of applications of this asymptotical theory is the study of the resonant drift of spiral waves, observed when the medium is subject to external forcing periodic in time, with the period close to the own period of the spiral wave $[4,5]$. Resonant drift has been suggested as a possible mechanism for low-voltage defibrillation devices. The idea is that since this phenomenon can be observed, at least in theory, for arbitrarily small magnitude of perturbation, it can be used to gently push the spiral waves out of fibrillating heart tissue. Currently used defibrillation method by 
single shock is not always effective, but can cause significant damage to cardiac tissue, and by far exceeds the pain threshold, even for implantable defibrillators.

The practical implementation of the resonant drift for defibrillation is stipulated by two kinds of conditions:

(i) Our ability to overcome 'imperfections' of the real heart as compared to the theory (inhomogeneity, complicated structure, etc). This led us to suggesting the feedback protocol of the resonant drift [6]. This protocol has been shown to work even in realistic complicated three-dimensional geometry of heart ventricles [7].

(ii) The realistic time required for elimination of spiral waves (say, shorter than $1 \mathrm{~min}$ ) at stimulation amplitude significantly less than that used for conventional single-shock defibrillation. This time is determined by the velocity of resonant drift. This velocity can be found by experiment, or numerical simulation with biophysically realistic models $[8,9,10]$. Both these ways are, however, rather complicated. The asymptotical theory provides, at least in principle, an easier way to estimate the velocity of resonant drift.

The method of response functions, based on the asymptotical theory, provides a more economical way to obtain answers to this and other analogous questions regarding 'soft' evolution of spiral waves. In this paper, this method is for the first time directly used to calculate the velocity of resonant drift. This is done for the Complex Ginzburg-Landau Equation, the first, and so far the only autowave system, for which response functions have been found explicitly [11]. This equation describes a self-oscillatory rather than excitable medium. Most of the electrically active tissues of the heart are excitable media. It was suggested that self-oscillatory behaviour of cardiac tissue may also play a role in fibrillation [12]. However, the purpose of the present study is development of the method, rather than immediate application to realistic models.

\section{The General Theory}

\subsection{REACTION-DIFFUSION SYSTEMS AND SPIRAL WAVES}

Consider reaction-diffusion system in two spatial dimensions, i.e. system of partial differential equations of the form

$$
\partial_{t} u=f(u)+D \nabla^{2} u+\epsilon h, \quad u, f, h \in \mathbb{R}^{\ell}, D \in \mathbb{R}^{\ell \times \ell}, \ell \geq 2 .
$$

where $u(\mathbf{r}, t)$ is a column-vector of reagent concentrations, $f(u)$ is a column-vector of reaction rates, $D$ is the matrix of diffusion coefficients, $\epsilon h(u, \mathbf{r}, t)$ is some small perturbation and $\mathbf{r} \in \mathbb{R}^{2}$ is the vector of coordinates on the plane. In cardiac tissue models, the role of reagents' concentrations is played by transmembrane voltage, gating variables of the channels and ionic concentrations, and the role of the diffu- 


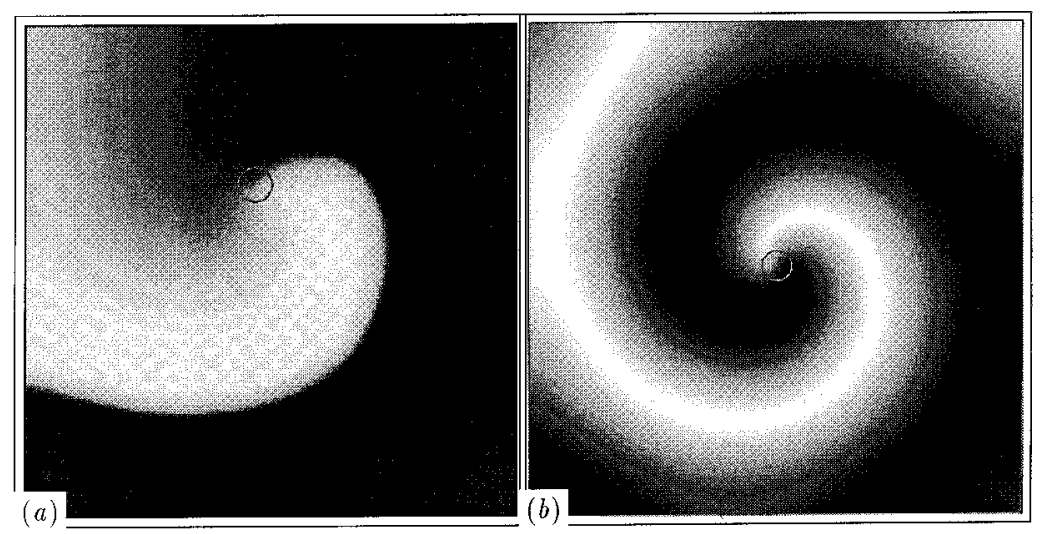

Figure 1. Spiral waves in different autowave systems. (a) In a model of rabbit atrial tissue [8]. Medium size $40 \times 40 \mathrm{~mm}$. The shading represents the transmembrane voltage $E$, lighter for depolarisation. The circle is the trajectory of the tip defined as intersection of isolines $E=-10 \mathrm{mV}$ and $\left[\mathrm{Ca}^{++}\right]_{i}=0.05 \mathrm{mM}$. (b) In CGLE (19), for $\alpha=0.5$ and $\beta=0$. Medium size $100 \times 100$ s.u. The shading represents $\operatorname{Re}(u)$, lighter for higher value. The circle is the trajectory of the tip defined as intersection of isolines $\operatorname{Re}(u)=0.9$ and $\operatorname{Im}(u)=0$.

sion is played by intercellular conductivity (thus, the only diffusing 'reagent' is the transmembrane voltage).

We assume that (1) has solutions in the form of steadily rotating spiral waves,

$$
u=U(\mathbf{r}, t)=U(\rho(\mathbf{r}), \vartheta(\mathbf{r})+\omega t) .
$$

Such solutions are observed in various reaction-diffusion systems; Figure 1 shows two examples. In some reaction-diffusion systems, including models of heart tissue, spiral waves do not rotate steadily, but meander. The case of steadily rotating spirals is much simpler and its theory is developed better.

Note that the unperturbed reaction-diffusion system, i.e. (1) with $\epsilon h=0$, has an obvious but important symmetry. Namely, it is invariant with respect to the Euclidean group of motions of the plane $\{\mathbf{r}\}$. Since solution (2) at any fixed $t$ is not invariant against this group, the group 'multiplies' this solution. That is,

$$
\tilde{U}=U(\rho(\mathbf{r}-\mathbf{R}), \vartheta(\mathbf{r}-\mathbf{R})+\Theta),
$$

where $\Theta=\omega t-\Phi$, is another solution for any constant displacement vector $\mathbf{R}=$ $(X, Y)^{\dagger}$ and initial rotation phase $\Phi$.

Thus, if the unperturbed system has one spiral wave solution, then it has a whole three-dimensional manifold of (relatively stable) such solutions.

\subsection{FINITE-DIMENSIONAL ANALOGY}

The asymptotic theory of drift of spiral waves [3] was proposed based on the analogy with finite-dimension problem of perturbation of an invariant manifold (see 


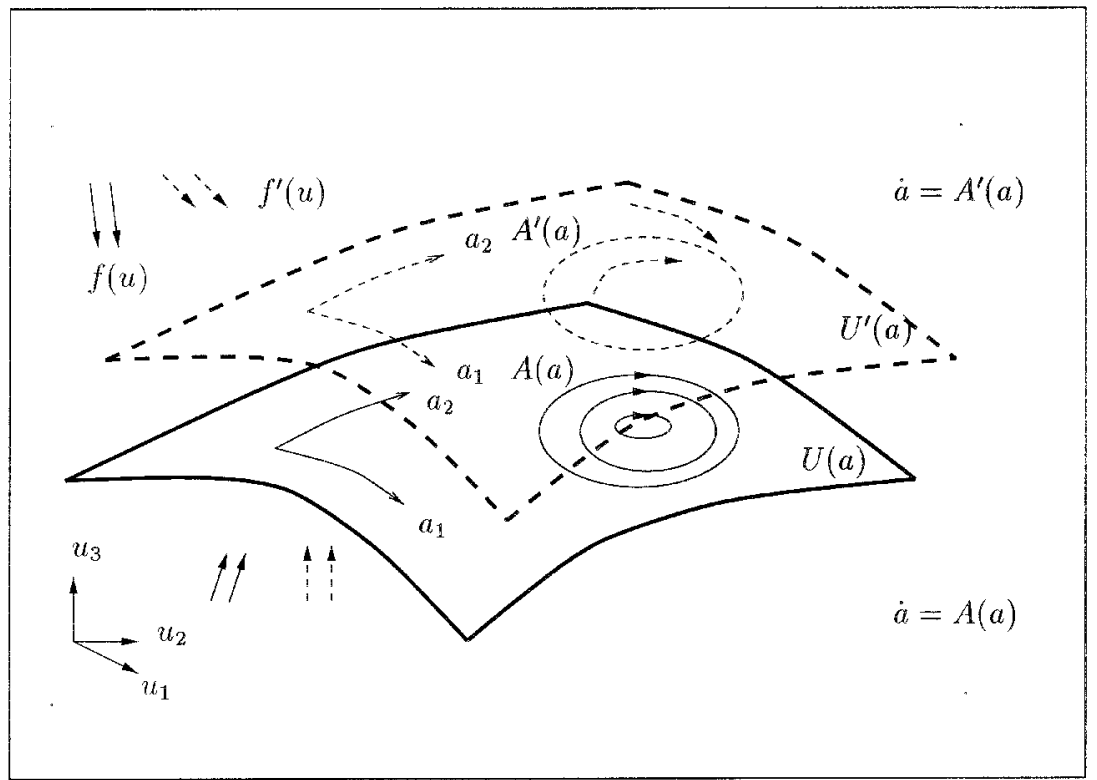

Figure 2. Perturbation of an invariant manifold. Vector field $f(u)$ in phase space with coordinates $u$ has an invariant manifold $U$ with coordinates $a$, and vector field $A$ on the manifold. Perturbed vector field $f^{\prime}(u)$ has a slightly different invariant manifold, $U^{\prime}$, and a slightly different vector field $A^{\prime}$ on it. Original objects are shown by solid lines, and perturbed objects by dashed lines.

Figure 2). If a vector field $f(u)$ in an $n$-dimensional phase space has an invariant $m$-dimensional manifold $U(a), m<n$, stable as a whole, then small perturbation of this vector field will, under certain conditions, preserve the invariant manifold, just slightly displacing it, $U \mapsto U^{\prime}$. Another effect of the perturbation is that the vector field on the shifted manifold $A^{\prime}(a)$ will be slightly different from the original one, $A(a)$. In practice, the existence of the original invariant manifold $U(a)$ could be due to a symmetry group. In that case, the flow on that manifold could be in some sense degenerated, and then the perturbation will remove this degeneracy.

To compare the two vector fields, on the original manifold and on the perturbed, we need to relate their coordinate systems $\{a\}$. A natural way is to require that the vector connecting two corresponding points $U(a)$ and $U^{\prime}(a)$, would not have a component along the manifold, i.e. along any of the tangent vectors $V_{j}(a)=$ $\partial U / \partial a_{j}$. In other words, it should be orthogonal,

$$
\left\langle W_{j}(a), U^{\prime}(a)-U(a)\right\rangle=0, \quad j=1 \ldots m
$$

to the projectors $W_{j}(a)$ onto the tangent vectors $V_{j}(a)$,

$$
\left\langle W_{j}(a), V_{k}(a)\right\rangle=\delta_{j, k} .
$$


These projectors are eigenvectors of the adjoint linearised matrix $(\partial f / \partial u)^{\dagger}(a)$. The two effects of the perturbation are produced by its two components, along and across the manifold, as determined by the projectors $W_{j}$.

If this finite-dimensional scheme can be applied to spiral waves, the role of the vector field is played by the reaction-diffusion system, so the phase space is a functional space. The invariant manifold is the three-dimensional manifold of spiral waves and is due to a symmetry group, the Euclidean group of the plane. The coordinates on the manifold are $\mathbf{R} \in \mathbb{R}^{2}$, the center of rotation of the spiral wave, and $\Theta$, its rotation angle. The flow on the manifold is degenerate, as it consists of relatively stable periodic orbits, which correspond to steady rotation of spiral waves around fixed centers:

$$
\Theta=\omega t-\Phi, \quad \Phi=\text { const } \quad \mathbf{R}=\text { const } .
$$

The perturbation removes this degeneracy, and we observe the drift of the spirals. By analogy with the finite-dimensional case, we expect that the flow on the manifold of spiral waves will be described by

$$
\partial_{t} \Theta=\omega+\epsilon F_{0}(\mathbf{R}, \Theta), \quad \partial_{t} \mathbf{R}=\epsilon \mathbf{F}_{1}(\mathbf{R}, \Theta),
$$

where $F_{0}$ and $\mathbf{F}_{1}$ are 'projections' of the perturbation onto the tangent space of the manifold. The right-hand sides of (7) depend on the phase $\Theta$. On the time scale $\epsilon^{-1}$ this phase oscillates fastly; averaging over these oscillations gives motion equations of the spiral waves,

$$
\partial_{t} \bar{\Theta}=\omega+\epsilon \bar{F}_{0}(\mathbf{R}), \quad \partial_{t} \bar{R}=\epsilon \overline{\mathbf{F}}_{1}(\mathbf{R}) .
$$

\subsection{RESPONSE FUNCTIONS}

Thus, the finite-dimensional analogy suggests that the dynamics of spiral waves (perhaps like that of many other dissipative structures) is described by 'Aristotelean' mechanics, when the velocity of motion is proportional to the applied perturbation. The right-hand sides in the equations, the 'forces', are projections of the perturbation onto the corresponding tangent space of the invariant manifold. This tangent space is a linear space, the span of the Goldstone modes, corresponding to the translations along the symmetry group, at $\mathbf{R}=\mathbf{0}$ and $\Theta=0$

$$
\begin{aligned}
V_{0} & =-\omega^{-1} \partial_{t} U(\mathbf{r}, t)=-\left.\partial_{\vartheta} U(\rho(\mathbf{r}), \vartheta(\mathbf{r}))\right|_{t=0}, \\
V_{ \pm 1} & =-\frac{1}{2} e^{\mp i \omega t}\left(\partial_{x} \mp i \partial_{y}\right) U(\mathbf{r}, t) \\
& =-\left.\frac{1}{2} e^{\mp i \vartheta}\left(\partial_{\rho} \mp i \rho^{-1} \partial_{\vartheta}\right) U(\rho(\mathbf{r}), \vartheta(\mathbf{r}))\right|_{t=0},
\end{aligned}
$$

which are critical eigenfunctions

$$
\tilde{L} V_{n}=i \omega n, \quad n=0, \pm 1,
$$


of the linearised operator

$$
\tilde{L}=D \nabla^{2}-\omega \partial_{\vartheta}+\left.\left(\frac{\partial f}{\partial u}\right)\right|_{u=U(\mathbf{r})} .
$$

Thus, for each particular point at the manifold, the projection operators map the functional space of the perturbations into the three-dimensional tangent space, and are thus just three functionals. Since all points of our manifold are equivalent to each other up to a Euclidean transformation of the plane, it is enough to know the projection functionals at one point. This symmetry consideration shows that if the functionals are written as integrals, they should have the form [3]:

$$
\begin{aligned}
\bar{F}_{n}(t)= & e^{i n \Phi} \oint_{t-\pi / \omega}^{t+\pi / \omega} \frac{\omega \mathrm{d} \tau}{2 \pi} \iint_{\mathbb{R}^{2}} \mathrm{~d}^{2} \mathbf{r} e^{-i n \omega \tau}\left\langle W_{n}(\rho(\mathbf{r}-\mathbf{R}), \vartheta(\mathbf{r}-\mathbf{R})\right. \\
& +\omega \tau-\Phi), h\rangle
\end{aligned}
$$

where

$$
h=h(U(\mathbf{r}, \tau), \mathbf{r}, \tau), \mathbf{R}=\mathbf{R}(t), \Phi=\Phi(t), \bar{F}_{1}=\left(\overline{\mathbf{F}}_{1}\right)_{x}+i\left(\overline{\mathbf{F}}_{1}\right)_{y},
$$

and kernels $W_{n}$ are eigenfunctions

$$
\tilde{L}^{+} W_{n}=-i \omega n W_{n}, \quad n=0, \pm 1
$$

of the adjoint linearised operator

$$
\tilde{L}^{+}=D \nabla^{2}+\omega \partial_{\vartheta}+\left.\left(\frac{\partial f}{\partial u}\right)^{+}\right|_{u=U(\mathbf{r})},
$$

corresponding to the Goldstone modes $V_{n}(9)$. We call these kernels response functions (RF).

Note that while the existence and even the explicit form of the Goldstone modes follows from the symmetry of the problem, the existence of the RF's does not, and is still an open question. In particular, this implies that equations (14) are formally overdetermined.

This is just one of quite a few delicate mathematical aspects of the applicability of the finite-dimensional scheme to the problem of the drift of spiral waves, which is not at all obvious. Paper [13] gives some flavour of related mathematical problems, mainly concerning relatively simple case of a Hilbert functional space on a bounded region. Dynamics of spiral waves apparently involves a more complicated case of a Banach space on the whole plane. If however, equations (14) have been solved, this analogy predicts that drift will be described by equations (8), (12). And a test of this prediction is a test of the viability of the asymptotic approach. 


\subsection{RESONANT DRIFT}

If the perturbation $h$ is homogeneous in space and periodic in time, $h(t) \equiv h(t+$ $2 \pi / \Omega$ ), with the frequency close to spirals own frequency of rotation, $\Omega \approx \omega$, then the equations of the drift reduce to [3]

$$
\partial_{t} \phi=\Omega-\omega-\epsilon H_{0}, \quad \partial_{t}(X+i Y)=\epsilon e^{i \phi} H_{1},
$$

where $\phi=\phi(t)$ is the instantaneous phase difference between the vortex orientation and the stimulation phase, $\phi=\Omega t-\bar{\Theta}$, and

$$
H_{n}=\left\langle\iint_{\mathbb{R}^{2}} W_{n} \mathrm{~d}^{2} \mathbf{r}, \oint e^{-i n \Omega \tau} h(\tau) \frac{\Omega \mathrm{d} \tau}{2 \pi}\right\rangle, \quad n=0,1 .
$$

This describes drift along a circle,

$$
X+i Y=X_{c}+i Y_{c}+R_{d} e^{i \delta t} ; \quad \delta=\Omega-\omega-\epsilon H_{0} ; \quad R_{d}=\epsilon H_{1} / \delta .
$$

If frequency difference $\delta$ is small or vanishes, the drift is along a circle of large radius or a straight line, the resonant drift [4].

This has a very simple 'physical' interpretation: if external perturbations occur at the same phase of the spiral wave, they cause its shifts in the same direction, next shift parallel to the previous. Thus, phenomenon of resonant drift is a consequence of the symmetry and is universal for all spiral waves.

\section{Application to CGLE}

\subsection{SPIRAL WAVES IN CGLE}

The Complex Ginzburg Landau Equation (CGLE) is a two-component reactiondiffusion system which occurs in various applications. In biophysical context, most interesting is that it describes the behaviour of the generic reaction-diffusion system after the reaction part has undergone Andronov-Hopf bifurcation [14]. Similar to the Hopf normal form, this equation is conveniently presented in the complex form:

$$
\partial_{t} u=u-(1-\ell \alpha) u|u|^{2}+(1+\ell \beta) \nabla^{2} u+\epsilon h,
$$

where $u \in \mathbb{C}, \alpha, \beta \in \mathbb{R}$. In this paper, we use $\alpha=0.5$ and $\beta=0$ (and omit $\beta$ ). The imaginary unit $\ell$ in this equation must be treated as a different mathematical entity from $i$ of the general theory. This is because (19) is, essentially, just a convenient form to write a symmetric system of real equations, rather than a truly complex equation, and the theory of [8] operates with real equations anyway: see [11] for more detail. Rigidly rotating spiral wave solutions to (19) have been studied by Hagan [15], and have the form

$$
U(\mathbf{r}, t)=e^{\ell(\vartheta+\omega t)} P(\rho)
$$


where $P(\rho)=a(\rho) e^{\ell \psi(\rho)} \in \mathbb{C}$ and $\omega$ solve a nonlinear eigenvalue problem

$$
\begin{aligned}
& P^{\prime \prime}+\frac{1}{\rho} P^{\prime}+\left(1-\ell \omega-(1-\ell \alpha)|P|^{2}-\frac{1}{\rho^{2}}\right) P=0, \\
& P(\rho \rightarrow 0) \propto \rho, \\
& P(\rho \rightarrow \infty) \approx \sqrt{1-k^{2}} \exp (\ell k \rho+o(\rho))(1+o(1)),
\end{aligned}
$$

$k$ is the asymptotical wavenumber and $\omega=\alpha\left(1-k^{2}\right)$. The response function have the form [11]:

$$
W_{n}=e^{(\ell-i n) \vartheta} Q_{n}(\rho)
$$

where $Q_{n}$ are solutions to linear problems

$$
\begin{aligned}
& Q_{n}^{\prime \prime}+\frac{1}{\rho} Q_{n}^{\prime}+\{1+\ell \omega \\
& \left.+\frac{(\ell-i n)^{2}}{\rho^{2}}-a^{2}\left[2(1+\ell \alpha)+(1-\ell \alpha) e^{2 \ell \psi} \mathcal{C}\right]\right\} Q_{n}=0 \\
& \left|Q_{n}(\rho \rightarrow 0)\right|<\infty, \quad Q_{n}(\rho \rightarrow \infty) \rightarrow 0 .
\end{aligned}
$$

As follows from the above, $W_{1}$ and $Q_{1}$ are 'bicomplex'-valued functions, each having four components, real and imaginary with respect to the two imaginary units $i$ and $\ell$. Thus to point to real or imaginary part of a quantity we have to specify which of the two imaginary units is meant.

Solutions to (23) were found numerically in [11], in the form

$$
\begin{aligned}
& Q_{0}(\rho)=(A(\rho)+\ell B(\rho)) e^{\ell \psi(\rho)}, \\
& Q_{1}(\rho)=(C(\rho)+\ell D(\rho)+i E(\rho)+i \ell F(\rho)) e^{\ell \psi(\rho)},
\end{aligned}
$$

where functions $C, D, E, F$ were tabulated. Corresponding response functions $W_{n}$ are illustrated in Figure 4. They are essentially nonzero only in the vicinity of the core of the spiral wave. This is the mathematical manifestation of the remarkable stability of spiral waves with respect to distant events.

\subsection{RESONANT DRIFT IN CGLE}

Let us consider perturbation

$$
h=\cos (\omega t),
$$

where $\omega$ is the rotation frequency of the free spiral wave. Substitution of $Q_{1}$ in (24) and (25) into (22), (16) and (17), with account of the normalisation (5), gives the following expression for the resonant drift velocity

$$
\left|\partial_{t} \mathbf{R}\right|=\epsilon\left|H_{1}\right|=\epsilon\left|\frac{\int_{0}^{\infty}[C-F+i(E+D)] e^{i \psi} \rho \mathrm{d} \rho}{2 \int_{0}^{\infty}\left[a F-\rho a^{\prime} C+i\left(a D-\rho a^{\prime} E\right)\right] \mathrm{d} \rho}\right| .
$$




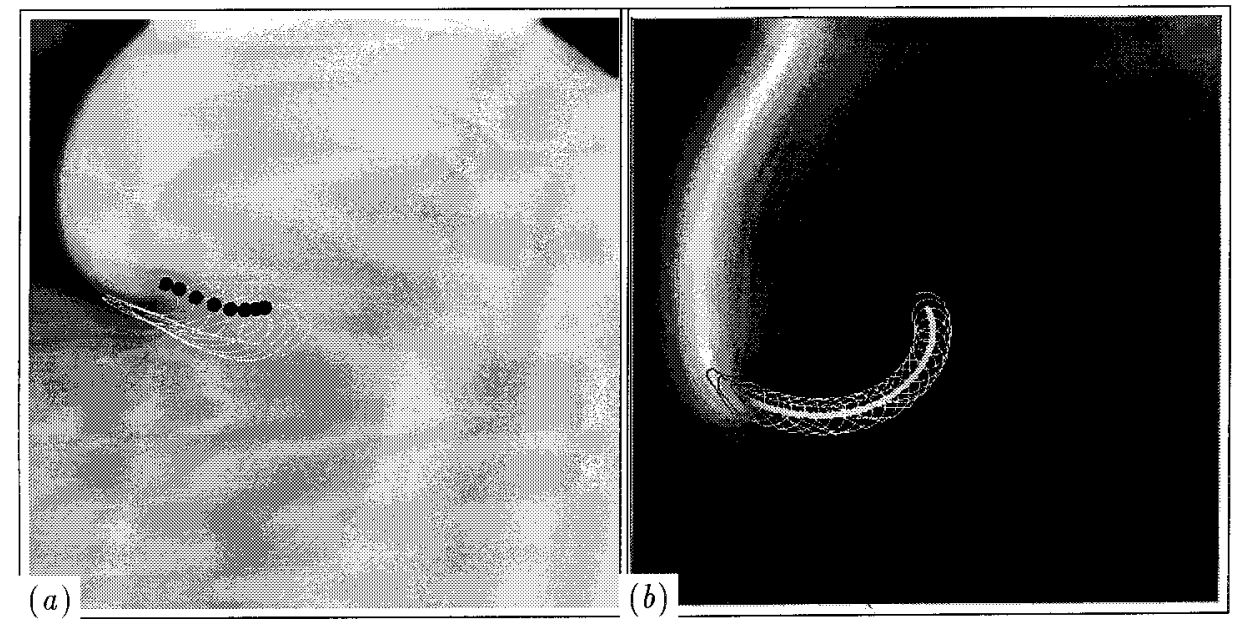

Figure 3. Resonant drift of spiral waves in different autowave systems. (a) In a model of guinea pig ventricular tissue [9]. Medium size $20 \times 20 \mathrm{~mm}$. The white line: trajectory of the tip, the black circles: positions of the tip at the same phases of the perturbation. (b) In CGLE (19), for $\alpha=0.5$ and $\beta=0$. Medium size $100 \times 100$ s.u., perturbation amplitude $\epsilon=0.05$, time step $h_{t}=0.025$ and space step $h_{x}=0.5$. The thin 'cycloidal' line: trajectory of the tip $u=0.9+0 \ell$, the thick line: trajectory of the center $u=0+0 \ell$.

Thus, if we know Hagan's solution, $a, \psi$ and the components of the response functions, $C, D, E, F$, equation (26) gives a theoretical prediction for the velocity of the resonant drift.

For $\alpha=0.5$ and $\beta=0$, functions $a, \psi, C, D, E, F$ were obtained numerically in [11]. By (26), this gives the normalised velocity $\left|\partial_{t} \mathbf{R}\right| / \epsilon=\left|H_{1}\right| \approx 2.8423$. This was calculated at spatial discretisation step $h_{\rho}=0.005$.

To check the theoretical prediction, we simulated CGLE (19) with perturbation (25) of amplitude $\epsilon$ up to 0.1. We used two finite-difference schemes, (i) firstorder explicit, and (ii) second-order alternating direction implicit, both with 5-point approximation of the Laplacian. Computational grid was of spatial size $100 \times 100$ to $300 \times 300$ s.u., with discretisation steps $h_{t}$ from 0.005 to 0.5 t.u. and $h_{x}$ from 0.2 to 0.5 s.u. Initial conditions were specified using Hagan's solution.

The trajectory of the center of the spiral was defined as the intersection of the null-isolines of $\ell$-real and $\ell$-imaginary parts of $u$. This trajectory was used to measure the velocity of the drift, with two different techniques. If the trajectories were long enough to form a circle, we fit them to (18) to find the velocity. For shorter pieces this was not possible, and then we used finite differencing with subsequent averaging. To reduce the influence of the small oscillations of the center with the period of the spiral wave rotation, we choose the differentiation steps as close to this period or its multiple as possible. We ensured that these two different techniques gave the same result within their natural precision, determined by the aforementioned oscillations of the center and influence of the space discretisation. 


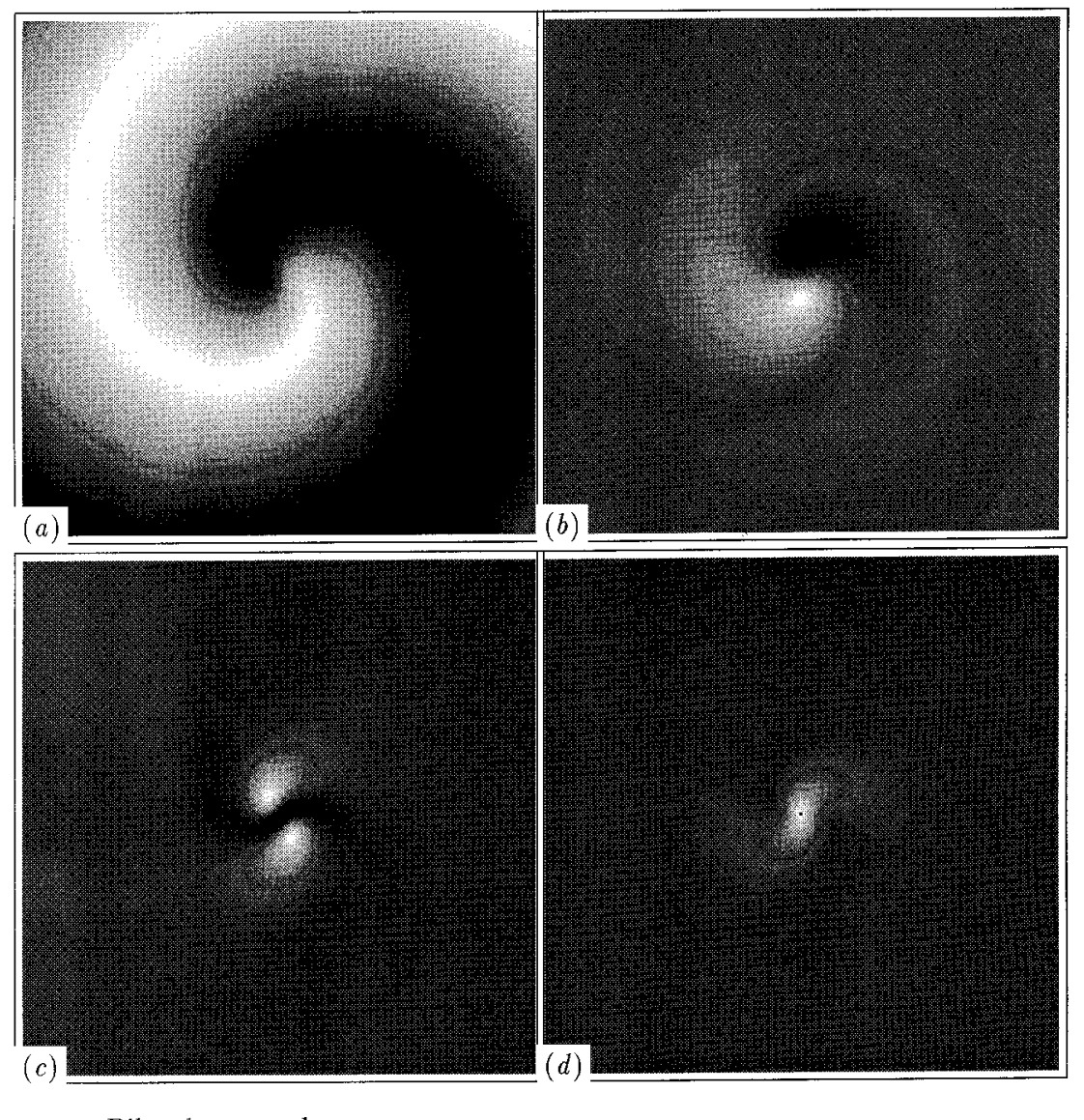

Figure 4. Response function of the spiral wave in CGLE [11]. (a) The spiral wave solution. (b) Temporal (rotational) RF. (c) $i$-real part ( $x$-direction) of spatial RF. (d) $i$-imaginary part ( $y$-direction) of spatial RF. Shown are $\ell$-real components, whereas $\ell$-imaginary components can be obtained by rotation of these by $90^{\circ}$. Higher value corresponds to lighter shade. Gray peripheries of $(b-d)$ correspond to zero. Size of the square is $60 \times 60$ s.u.

Some typical trajectories are shown at Figure 3(b) and Figure 5. Interaction with boundaries is seen only at distances less than 30 s.u. At further distances, and for time exceeding some transient, the trajectories of the centre are approximately circular. The radii of these circles decreased, and the velocity of the drift increased with the perturbation amplitude. Both these qualitative observations are in agreement with theory.

In the agreement with theory, the velocity of the resonant drift was approximately proportional to the perturbation amplitude $\epsilon$, see Figure 6 . Note that this proportionality is obeyed quite well even for $\epsilon=0.05$, and that at this amplitude the drifting spiral wave is considerably deformed, see Figure 3(b). This is because the deformation is due to the relative motion ('Doppler effect' [16]), which affects 


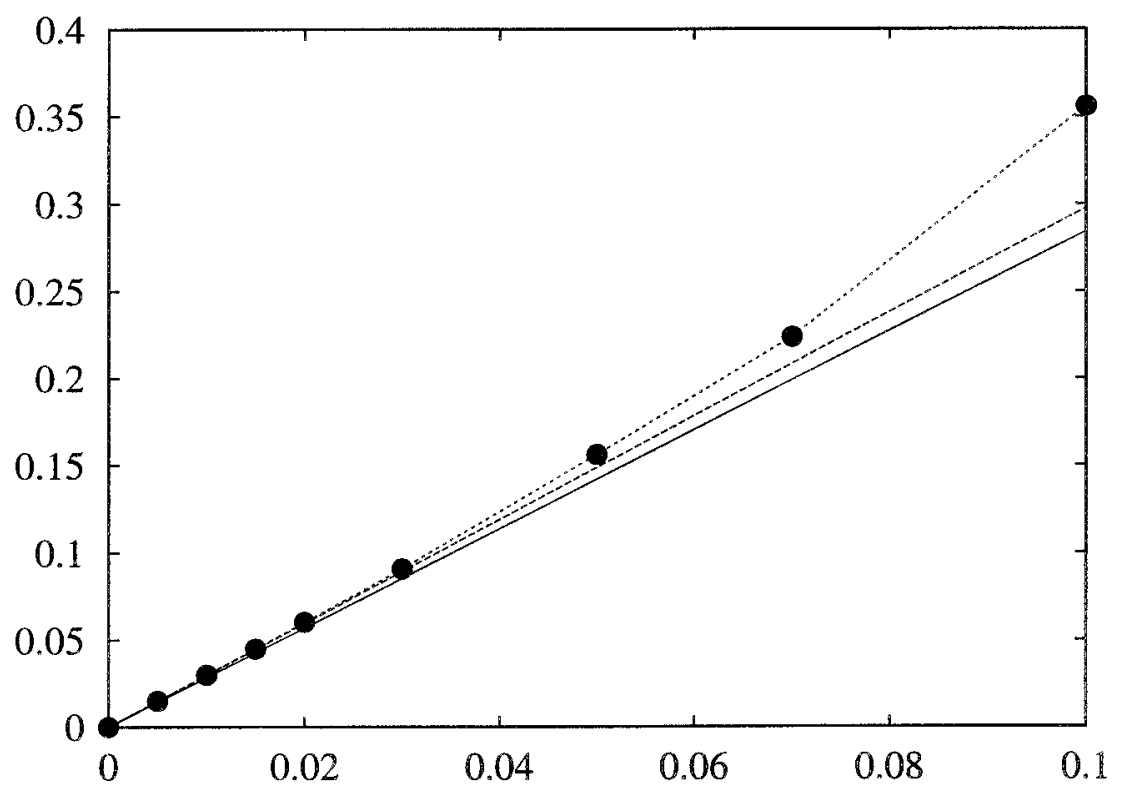

Figure 5. Trajectories of resonant drift at different $\epsilon$ (shown by labels at the trajectories), medium $100 \times 100$ s.u., $h_{t}=0.025, h_{x}=0.5$.

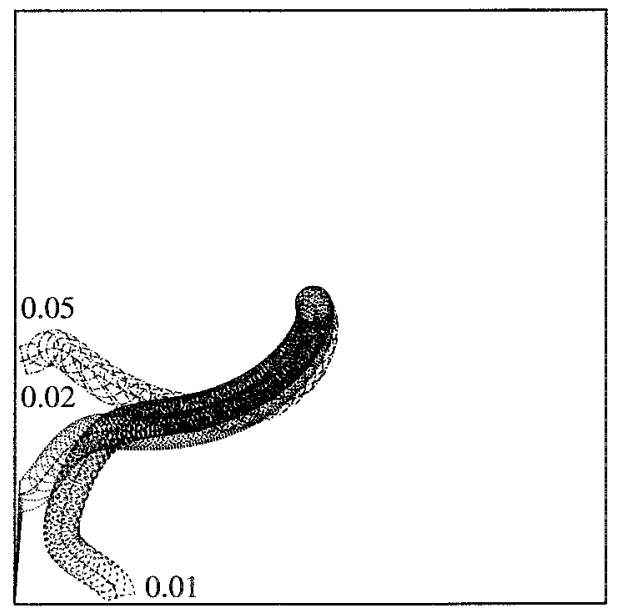

Figure 6. Drift velocity $\left|\partial_{t} \mathbf{R}\right|$, as a function of the perturbation amplitude $\epsilon, h_{t}=h_{x}=0.5$. Dotted line with points: numerical results. Solid line: theoretical dependence. Dashed line: best linear fit of numerical results at smaller amplitudes. 


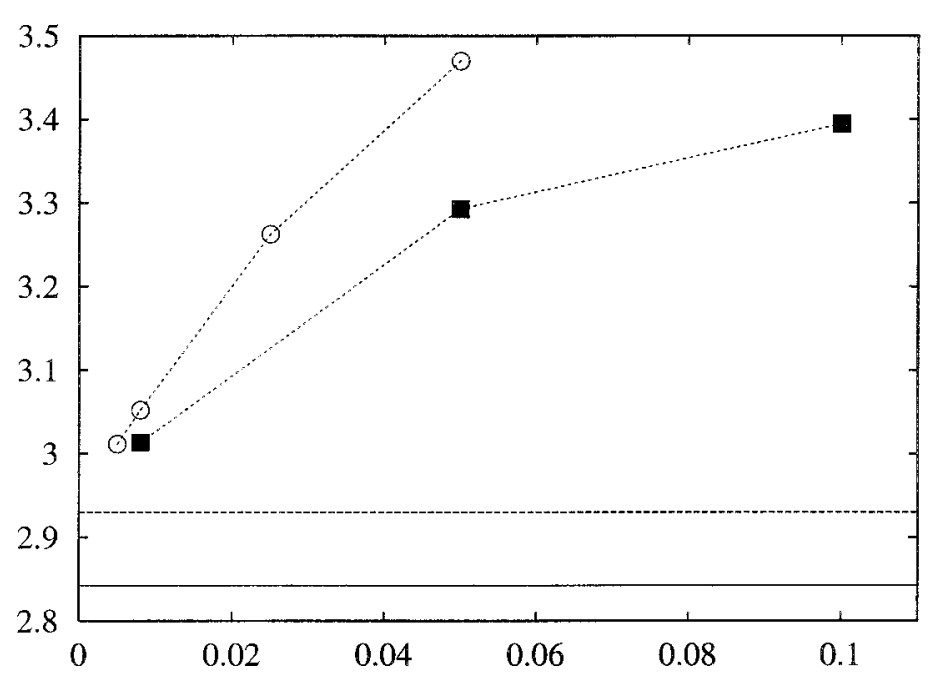

Figure 7. Normalised drift velocity, $\left|\partial_{t} \mathbf{R} / \epsilon\right|$, as a function of time step $h_{t}$, at perturbation amplitude $\epsilon=0.01$. Dotted line with open circles: explicit scheme, space step $h_{x}=0.5$. Dotted line with filled squares: ADI scheme, space step $h_{x}=0.2$. Solid line: theoretical value $2.84 \ldots$. . Dashed line: 'coarse-grained' theoretical value $2.93 \ldots$. estimated for radial step 0.2 .

the periphery of the spiral, whereas the velocity of the drift is determined by the events in the core where the response functions are nonzero.

To check the quantitative predictions, we measured the normalised drift velocity, $\left|\partial_{t} \mathbf{R} / \epsilon\right|$ in numeric simulations, and its behaviour as $\epsilon \rightarrow 0, h_{t} \rightarrow 0$ and $h_{t} \rightarrow 0$. The results are shown on Figure 7 .

The crucial parameter limiting the convergence to the theoretical value, was the spatial discretisation step of the numerical simulation. To check how important this parameter is, we calculated the theoretical value with a precision adequate to the that of the numerical simulations. Namely, we repeated the calculations of the Hagan's solution and the response functions, i.e. solved the systems (21), (23), with step in $\rho$ equal to the smallest discretisation step used in our simulations, 0.2 s.u. And the integrals of (26) were then estimated by the trapezoidal rule, which corresponds to the approximation order of the PDE simulation. The resulting 'coarse-grained' theoretical value was $\left|H_{1}\right| \approx 2.93$ and is also shown on Figure 7. It can be seen that at smallest steps used in simulations, the difference from the theoretical value is comparable to the error introduced by coarse-graining in the theoretical value itself. Thus, predictions of the asymptotical theory agree qualitatively and quantitatively with the results of direct numerical simulations, up to the precision achievable by these simulations. 


\section{Conclusions}

- Up to now, the resonant drift has been studied in excitable media. In this paper we have shown it in CGLE, a model of an oscillatory rather than an excitable medium. The general qualitative properties of the resonant drift in this model are the same as in excitable systems, and as predicted by the general asymptotical theory.

- We used the response functions to predict quantitatively the properties of the resonant drift, e.g. its velocity. This predictions are in a good agreement with direct numerical simulations.

- Thus, the asymptotical theory of spiral wave dynamics [3] has been, for the first time, confirmed directly and quantitatively. This shows that, despite all assumptions made by the theory, it gives correct predictions.

- The methods of response functions could be used to predict the dynamics of spiral and scroll waves in models of practical interest, by a computationally less expensive way than direct numerical simulation. In the case of CGLE it is solution of 1D systems of equations; in general case it would be 2D systems, as opposed to $2 \mathrm{D}+$ time or $3 \mathrm{D}+$ time of direct numerical simulations.

\section{Acknowledgements}

We are grateful to Prof. A.V. Holden for the kindly provided possibility to fulfill the computational part of this work in his laboratory, and for valuable linguistic and stylistic advice. This work has been supported in part by grants from Russian Foundation for Basic Research (96-01-00592) and Wellcome Trust (045192).

\section{References}

1. Gray, R.A. and Jalife, J.: Spiral waves and the heart, Int. J. of Bifurcation and Chaos 6 (1996), 415-435.

2. Keener, J.P.: The dynamics of 3-dimensional scroll waves in excitable media, Physica D 31 (1988), 269-276.

3. Biktashev, V.N. and Holden, A.V.: Resonant drift of autowave vortices in $2 \mathrm{D}$ and the effects of boundaries and inhomogeneities, Chaos, Solitons and Fractals 5 (1995), 575-622.

4. Davydov, V.A., Zykov, V.S., Mikhailov, A.S. and Brazhnik, P.K.: Drift and resonance of spiral waves in active media, Radiofizika 31 (1988), 574-582.

5. Agladze, K.I., Davydov, V.A. and Mikhailov, A.S.: Observation of a helical wave resonance in an excitable distributed medium, JETP Lett. 45 (1987), 767-770.

6. Biktashev, V.N. and Holden, A.V.: Design principles of a low-voltage cardiac defibrillator based on the effect of feed-back resonant drift, J. Theor. Biol. 169 (1994), 101-113.

7. Holden, A.V.: Defibrillation in models of cardiac muscle, J. Theoretical Medicine 1 (1997), 91-102.

8. Biktashev, V.N. and Holden, A.V.: Control of re-entrant activity in a model of mammalian atrial tissue, Proc. Roy. Soc. London B 260 (1995), 211-217. 
9. Biktashev, V.N. and Holden, A.V.: Re-entrant activity and its control in a model of mammalian ventricular tissue, Proc. Roy. Soc. London B 263 (1996), 1373-1382.

10. Biktashev, V.N. and Holden, A.V.: Re-entrant waves and their elimination in a model of mammalian ventricular tissue, Chaos 8 (1998), 48-56.

11. Biktasheva, I.V., Elkin, Yu.E. and Biktashev, V.N.: Localised sensitivity of spiral waves in the Complex Ginzburg-Landau Equation, Phys. Rev. E 57 (1998), 2656-2659.

12. Noble, D., Leguennec, J.Y. and Winslow, R.: Functional roles of sodium-calcium exchange in normal and abnormal cardiac rhythm, Ann. N. Y. Acad. Sci. 779 (1996), 480-488.

13. Robinson, J.C.: Finite-dimensional behavior in dissipative partial differential equations, Chaos 5 (1995), 330-345.

14. Kuramoto, Y. and Tsuzuki, T.: On the formation of dissipative structures in reaction-diffusion systems: Reduction perturbation approach, Prog. Theor. Phys. 54 (1975), 687-699.

15. Hagan, P.S.: Spiral waves in reaction-diffusion equations, SIAM J. Appl. Math. 42 (1982), 762 786.

16. Wellner, M., Pertsov, A.M. and Jalife, J.: Spatial Doppler anomaly in an excitable medium, Phys. Rev. E 54 (1996), 1120-1125. 